\title{
Trivium
}

Revue franco-allemande de sciences humaines et sociales - Deutsch-französische Zeitschrift für Geistesund Sozialwissenschaften

33 | 2021

Concepts historiques fondamentaux - Démocratie

\section{Introduction : fondements antiques}

\section{Christian Meier}

Traducteur : Anthony Andurand

\section{(2) OpenEdition}

\section{Journals}

Édition électronique

URL : https://journals.openedition.org/trivium/7620

DOI : $10.4000 /$ trivium. 7620

ISSN : 1963-1820

Éditeur

Les éditions de la Maison des sciences de l'Homme

Référence électronique

Christian Meier, «I. Introduction : fondements antiques », Trivium [En ligne], 33 | 2021, mis en ligne le 07 juin 2021, consulté le 10 juin 2021. URL : http://journals.openedition.org/trivium/7620 ; DOI : https://doi.org/10.4000/trivium.7620

Ce document a été généré automatiquement le 10 juin 2021

\section{(c) $(1)(9)$}

Les contenus des la revue Trivium sont mis à disposition selon les termes de la Licence Creative Commons Attribution - Pas d'Utilisation Commerciale - Pas de Modification 4.0 International. 


\title{
I. Introduction : fondements antiques
}

\author{
Christian Meier
}

Traduction : Anthony Andurand

\section{NOTE DE L'ÉDITEUR}

\section{p. 821-835 de l'article original}

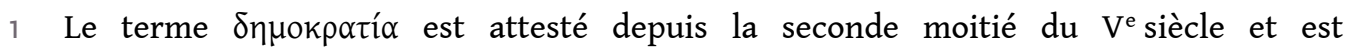

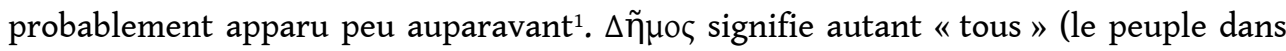
son ensemble, en particulier à l'assemblée) que «le grand nombre» (la foule, la majorité habituelle de l'assemblée). Selon le point de vue, le terme peut être compris de manière positive ou négative ${ }^{2}$. C'est peut-être pour des motifs extérieurs que la domination [Herrschaft] du peuple a été désignée au moyen du suffixe -kpatía, à la différence de la construction utilisée pour les concepts plus anciens de $\mu$ ov- $\alpha \rho x i ́ \alpha$ et d'ỏ $\lambda r \gamma-\alpha \rho x i^{\prime} \alpha^{3}$. À Athènes et ailleurs très certainement, avant l'apparition du terme de $\delta \eta \mu о к \rho \alpha \tau i ́ \alpha$ (et souvent encore par la suite, comme chez Aristote), on a désigné la domination du peuple au moyen du terme $\delta \tilde{\eta} \mu \circ \varsigma^{4}$.

2 La distinction des constitutions en fonction du détenteur du pouvoir [Herrschaft] - et ainsi de trois constitutions - apparaît pour la première fois dans une ode composée vers 470 par Pindare : « [...] en tout pays (vónoc), auprès des tyrans, là où règne la foule impétueuse [= les citoyens (hoplites)], et dans les cités que régissent les sages ${ }^{5} »$. Bien auparavant, on savait naturellement que le pouvoir dans la polis pouvait être réparti de différentes manières. [822] Ce constat résultait simplement de la présence de la tyrannie, distincte de la forme normale de la communauté dirigée par l'aristocratie. Les

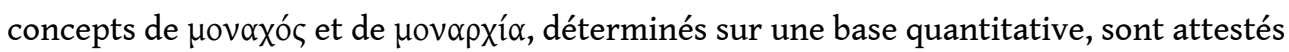
au VI siècle. Chez Homère, déjà, le "pouvoir d'un grand nombre de chefs » (de plusieurs aristocrates) apparait comme l'antithèse négative du « chef unique ${ }^{6}$ ». Mais si

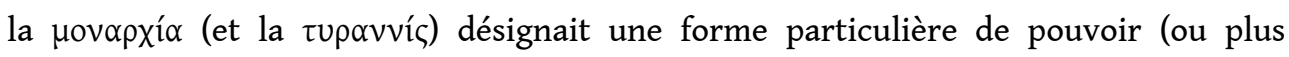


précisément de position de pouvoir ${ }^{7}$ ) et si celle-ci déterminait l'état global d'une polis, rien n'indique pourtant que l'état de la cité en question était déjà conçu et décrit, à l'époque, comme une "tyrannie", ou que l'on distinguait déjà deux sortes d'organisation politiques sur la base du critère de la domination. Ce n'est qu'au $\mathrm{V}^{\mathrm{e}}$ siècle que l'on découvrit que les constitutions devaient être considérées comme des formes de domination (d'un seul, d'un petit nombre, d'un grand nombre, de tous ...). Comme cette découverte fut décisive dans la genèse du concept de "démocratie " comme pour la classification des constitutions dans son ensemble, nous devons présenter ici ses origines et ses présupposés.

3 À l'époque archaïque, la loi (vónoc) constituait un critère déterminant pour caractériser l'organisation d'une communauté. La seule désignation positive d'une telle organisation que l'on rencontre au VIe siècle est celle d'Eủvouí $\alpha$ (soit le « bon ordre » en général) $)^{8}$. Le terme renvoyait alors, dans toute la Grèce, à la « constitution » des temps anciens, reposant sur un vópoৎ d'ordre religieux (« coutume », « loi », « origine ») et perçue comme un idéal normatif. Selon la problématique de cette époque, il ne s'agissait pas tant que le vónos fût bon, mais qu'il s'appliquât véritablement. Le modèle fondamental d'une communauté façonnée dans une certaine mesure par une logique patriarcale était déjà plus ou moins élaboré. Le pouvoir - quel qu'il fût - était presque partout détenu par la noblesse. Des différences sociales et institutionnelles existaient, mais elles n'étaient manifestement pas suffisamment marquées pour que l'on fût conduit à distinguer différentes sortes d'organisations politiques. L'Ev่vouía ne connut

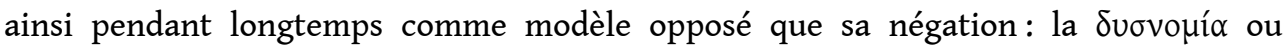
l'óvouía (le "désordre ", le "dérèglement»). La question centrale, pour évaluer et caractériser l'état et, partant, la constitution d'une communauté résidait dans ses dispositions en matière d'application de la loi. Et dans la mesure où par «loi » on entendait partout peu ou prou la même chose, seules deux réponses étaient possibles.

4 La question de la répartition du pouvoir [Herrschaft] se résumait largement à cela et était, par ailleurs, secondaire. Aux yeux de la strate influente de la noblesse, la tyrannie apparaît la plupart du temps comme une domination (et une servitude) et par conséquent comme une déviance par rapport à l'ordre légal normal (qui ne pouvait la concevoir comme une forme particulière de domination). Elle la rangeait - tout comme, probablement, les régimes aristocratiques qui fonctionnaient mal - parmi les dysnomies. D'autres ont pu concevoir la tyrannie comme une eunomie, mais on peut se demander si cette conception, si tant est qu'elle ait existé, a pu jamais dépasser le cercle des partisans des tyrans.

5 Cependant, dans la mesure où l'zủvouía, en tant que référence et idéal, [823] présupposait le bon vieux droit, son caractère contraignant était tributaire de ce que la compréhension du vópos fût, dans une certaine mesure, générale et par conséquent objective. Comme ce point suscita certainement toujours des vues différentes, cette référence tendit à s'effacer dans la mesure où les classes [Stände] et les communautés se différenciaient progressivement. Ce n'est toutefois que vers 500 qu'un changement significatif semble être apparu. Un nouvel idéal prit alors largement le relais de l'ancien: l'íoovouía (à savoir l'« ordre fondé sur l'égalité des droits civiques»). Une partie du peuple qui n'appartenait pas à l'aristocratie, d'abord surtout des paysans, mais ensuite aussi, fréquemment, les strates les plus basses, réclamèrent l'égalité des droits politiques - pas nécessairement l'accès aux magistratures, mais l'accès à l'assemblée, qui était investie ou qu'il fallait investir d'importantes fonctions, au 
tribunal du peuple, qu'il fallait souvent commencer par constituer et la plupart du

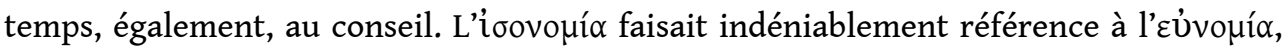
mais n'entrait pas nécessairement en opposition et devait seulement modifier, sans doute, l'ancien idéal : de plus larges parts de la communauté civique s'autonomisaient politiquement et prenaient, au nom des bonnes vieilles lois', le contrôle de la communauté. Les droits devaient être identiques, non seulement au nom de l'égalité, mais aussi au nom du droit lui-même, que la majorité des citoyens - y compris de nombreux aristocrates - était le mieux à même de garantir. Tout compte fait, l'íøovouía répondait à la question de savoir comment les droits politiques étaient ou devaient être répartis et devint ensuite presque interchangeable avec la $\delta$ nок $\rho \alpha \tau^{\prime} \alpha^{10}$.

Il est seulement possible d'esquisser le cheminement par lequel on en vint ensuite à l'idée que ce nouveau régime constituait une "domination du peuple», mais surtout qu'il devait être essentiellement décrit comme tel. Il se peut que les tenants de ce régime aient encore pensé un temps qu'il se caractérisait par ceci qu'il consolidait les droits. Ils voyaient dans la position de l'assemblée ( $\delta \tilde{\eta} \mu \circ \varsigma)$ leur garantie institutionnelle. Celle-ci fut placée à Athènes sous la protection de dispositions particulières (de telle sorte que, à mesure que les conditions de la nouvelle organisation furent comprises de manière plus englobante, cette compréhension put avec le temps être transposée sur le terme $\delta \tilde{\eta} \mu o \varsigma$, jusqu'à ce que celui-ci revêtit le sens de "domination du peuple $\left.{ }^{11} »\right)$. On affirma très tôt, volontairement et avec fierté, qu'en

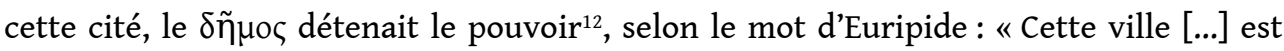
libre; le peuple y commande à son tour ${ }^{13} »$. La plupart du temps, on insistait ainsi c'est également le cas dans l'historiographie - sur le contraste avec la tyrannie, de sorte qu'il semblait ne rien exister d'autre, aucun troisième terme qui ne soit ni l'íøovopía (ou la $\delta \eta \mu о к \rho \alpha \tau i ́ \alpha)$, ni la tyrannie. Cela ne saurait signifier que tous les régimes qui n'étaient pas tyranniques étaient considérés comme «isonomiques» ou "démocratiques ", mais seulement qu'à Athènes et surtout ailleurs, on regardait la tyrannie comme un contre-modèle (et que l'on négligeait à l'inverse les régimes strictement aristocratiques). Dans la perspective de la domination du peuple, l'opposition essentielle n'en demeurait pas moins l'opposition entre régimes « isonomiques » et régimes «non isonomiques ${ }^{14}$ ». Dans les régimes isonomiques, la communauté des citoyens, dans son ensemble ou pour une part relativement importante, formait l'instance politique suprême. [824] Dans ces régimes, la cité est ainsi $\alpha$ Ủ première fois dans l'histoire universelle - l'on fût convaincu de posséder à travers ce régime des garanties institutionnelles en matière de droit joua un rôle tout particulier, tandis qu'auparavant et ailleurs, presque tout dépendait du caractère de ceux qui gouvernaient ${ }^{16}$. Il ne semble pas y avoir eu une opposition aux aristocrates et à leurs conceptions, si l'on ne tient pas compte du rejet de groupes particulièrement autoritaires et de leur gouvernement.

7 Dans la conception générale au sein de l'iøovouí $\alpha=\delta \eta \mu$ possibilité sur le plan légal, on ne distinguait au fond - comme au temps de l'idéal de l'zủvouía - que deux sortes de dominations politiques. Le règne du $\delta \tilde{\eta} \mu о \varsigma$ apparaissait comme l'unique forme légitime de domination : «toute décision y est portée devant le peuple [...], car seul compte l'ensemble ${ }^{17} »$. De même que l'égalité civique apparaissait

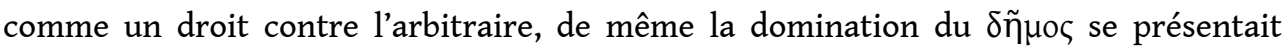
comme une liberté contre le despotisme des tyrannies et des oligarchies fondées sur 


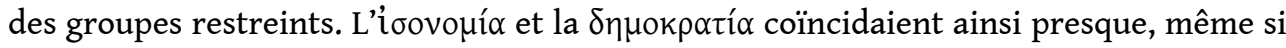
l'iøovouía ne désignait pas fondamentalement un type spécifique de constitution, mais d'abord un étalon à l'aune duquel les constitutions pouvaient être évaluées ${ }^{18}$. La démocratie, cependant, satisfaisait précisément à cet étalon.

Les choses se présentaient autrement aux yeux des partisans des anciennes formes aristocratiques: là, on se trouvait pris entre la démocratie et la monarchie. La classification fondée sur trois types de constitutions s'imposait tout simplement ${ }^{19}$. Ce n'est donc peut-être pas un hasard si on la rencontre pour la première fois chez Pindare. De même, la théorie des constitutions introduite au milieu du Ve siècle ne put que prendre conscience de ce triptyque ${ }^{20}$. La noblesse envisageait sa propre domination comme légitime, tandis que - conformément à sa vision du $\delta \tilde{\eta} \mu \circ \varsigma$ - elle considérait le gouvernement du peuple comme le « règne de la populace». L'aristocratie considérait sa propre domination comme légitime, alors que selon l'idée qu'elle se faisait du $\delta \tilde{\eta} \mu \circ \varsigma$, elle considérait la domination exercée par le peuple comme le «régime de la

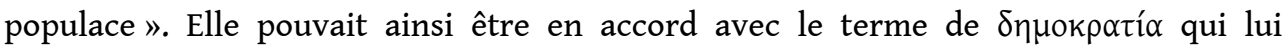
permettait de démasquer l'íoovouía (comme à l'inverse les opposants de l'aristocratie

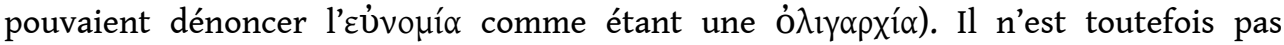

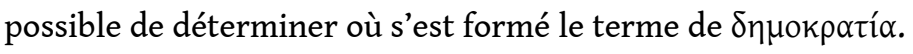

Le concept de $\delta \eta \mu о к \rho \alpha \tau i ́ \alpha$ fut exacerbé et fit l'objet d'une compréhension nouvelle, plus profonde, vers le milieu du $\mathrm{V}^{\mathrm{e}}$ siècle. À l'époque, une nouvelle forme de politique spécifiquement démocratique était apparue à Athènes, qui avait été définie en partie [825] en opposition à l'oligarchie spartiate et plus globalement en rupture avec les anciennes normes communes à l'ensemble des Grecs. Cette politique fut rendue possible par la participation systématique des strates inférieures à la vie et aux affaires de la communauté. Dans le sillage de cette évolution, deux positions relativement

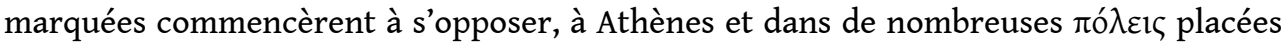
sous son influence ${ }^{21}$. Les classes moyennes (surtout celles de la campagne) ne réclamèrent pas d'être prises en compte. Au sein d'une large majorité du peuple, aucune différence d'opinion et de position ne pouvait dans les faits se faire jour, du fait des capacités intellectuelles du simple citoyen, de l'organisation politique et, en conséquence, de l'absence de points de cristallisation sur les sujets politiques. D'une manière générale, le "peuple " avait coutume de réagir comme un bloc ${ }^{22}$ et avait fait corps, précisément, avec une politique déterminée. Cependant, cela voulait dire que les nobles, pour autant qu'ils voulussent faire prévaloir une politique conforme à leurs normes, à leurs conceptions et aux liens qu'ils entretenaient, étaient régulièrement et nécessairement contraints de s'incliner dans la plupart des démocraties. La mise en place d'une forme constitutionnelle démocratique et la poursuite d'une politique déterminée allaient généralement de pair. Bien souvent, du fait de l'étroite imbrication de la politique intérieure et de la politique extérieure, un changement dans les orientations politiques présupposait ou bien se traduisait par un changement de constitution $^{23}$. De manière symptomatique, l'animosité entre les différentes classes [Stände] était souvent si forte - ou bien la cohésion politique était souvent si faible que, à la guerre, l'on se sentait plus proche des sympathisants dans les autres cités que des adversaires au sein de la même cité.

Les conséquences de ces évolutions se manifestent pour nous pour la première fois dans le pamphlet rédigé vers 430 par un aristocrate athénien, le Pseudo-Xénophon. Selon lui, la démocratie attique consacrait la domination du bas peuple. Ce dernier était 
vu comme pauvre, ignorant et frénétique ; sa politique était mauvaise, mais conforme à

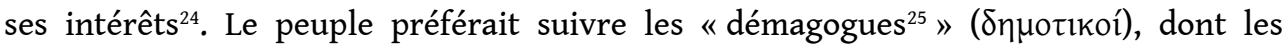
conseils résultaient de l'ignorance, de la perversité et de la flagornerie, que des hommes «bons et utiles ${ }^{26}$ » faisant preuve de vertu, de raison et d'hostilité à son encontre.

Le Pseudo-Xénophon percevait ainsi un clivage fondamental et profond entre l'aristocratie et le peuple. Les intérêts, les opinions et la politique tout entière de chacun de ces groupes étaient diamétralement opposés. Par conséquent, la démocratie ne pouvait en aucun cas être significativement améliorée: elle ne pouvait que se maintenir ou disparaitre ${ }^{27}$. Avec une certaine distance, le pamphlet concevait ainsi le contenu politique comme un trait caractéristique de la démocratie athénienne. Les observations formulées au sujet d'Athènes sur un ton polémique devaient ensuite être exprimées dans une perspective plus générale dans la théorie politique d'Aristote. Le fait que le pouvoir suprême, écrit en effet ce dernier, [826] soit aux mains du plus grand nombre dans la démocratie (et de quelques-uns dans l'oligarchie) est fortuit. La véritable différence entre les différents groupes détenteurs du pouvoir réside dans l'opposition entre riches et pauvres ${ }^{28}$. Dans d'autres passages, des considérations plus nuancées l'amènent à la conclusion qu'il y aura démocratie dès lors qu'« une majorité de gens libres mais modestes seront les maîtres du pouvoir ${ }^{29}$ ». Il est vrai que la discussion politique se déplaçait d'ores et déjà dans le domaine économique, de sorte que l'effacement partiel des clivages généraux qui prévalaient jusqu'alors en matière de politique extérieure était compensé par le maintien des oppositions entre les différents groupes [Stände], devenues des oppositions de classes.

Toutefois, à mesure que l'on reconnaissait une divergence d'intérêts fondamentale et tout à fait fondée entre l'aristocratie et les masses, une conception plus globale, définie politiquement, de la constitution se développa. Tant que l'on ne tenait le peuple que pour ignorant et frénétique ${ }^{30}$, il restait somme toute possible de le manœuvrer. Désormais, on découvrit - ou bien l'on admit - que non seulement les institutions, le cadre des droits civiques et la politique, mais aussi la manière de penser et de vivre, la nature de la vertu civique et des lois n'étaient pas les mêmes dans les différents régimes $^{31}$. La répartition du pouvoir était si prédominante qu'elle semblait définir la communauté dans son ensemble. Dès lors la question de savoir quels étaient les citoyens actifs revêtait une nouvelle signification : elle déterminait souvent la manière dont on était gouverné et dont on vivait. Le degré et l'extension des droits civiques

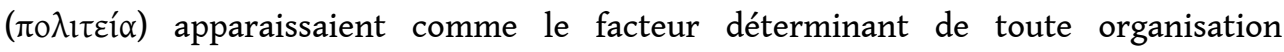
politique.

C'est ainsi que l'on envisagea les diverses constitutions non pas tant en fonction de leurs institutions ou selon qu'elles reposaient sur la domination d'un seul, de quelques-

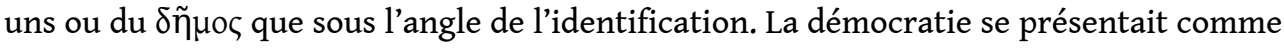

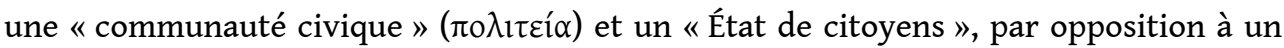

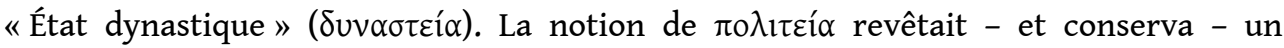
contenu normatif, mais fut très vite formalisée pour désigner une organisation politique $^{32}$. Ce n'est pas uniquement par ironie qu'il fit son apparition au moment où l'on apprit à distinguer de manière plus marquée le bien de la cité (= la

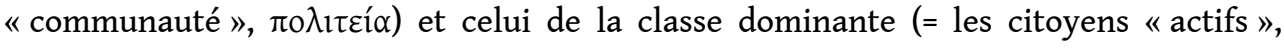

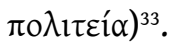


14 Avec la conceptualisation essentiellement politique des « communautés civiques » (= les constitutions), autrement dit d'un nouveau type d'oppositions politiques, une nouvelle classification des régimes politiques se développa presqu'au même moment : plusieurs régimes, qui jusqu'alors étaient considérés comme des démocraties, [827] étaient davantage perçus, dorénavant, comme des régimes oligarchiques ou mixtes, à l'instar de nombreuses démocraties hoplitiques, conservatives dans leur contenu ${ }^{33 a}$. La question de l'égalité se posait elle aussi sous un jour nouveau : dans un premier temps, le citoyen pauvre avait voulu être l'égal du citoyen le plus éminent; désormais, les citoyens les plus éminents, en tant que minorité, devenaient souvent, pour ainsi dire, des citoyens pénalisés ${ }^{34}$. Et comme il était dorénavant clair, pour de larges groupes, que les démocraties ne faisaient pas s'opposer la majorité à un seul ou à quelques-uns, mais un groupe de la communauté contre un autre, la classification tripartite des constitutions acquit tout son poids. Plus la domination du peuple était envisagée comme étant conditionnée par des intérêts, moins une alternative légale à celle-ci pouvait être contestée.

À la même époque, peu ou prou, il apparut clairement que la tripartition des constitutions était insuffisante. En se référant probablement à l'ancien idéal de l'iøovouía, on chercha à esquisser l'idée nouvelle d'une constitution véritablement équitable et commune ${ }^{35}$, une manière de constitution mixte, envisagée comme le retour

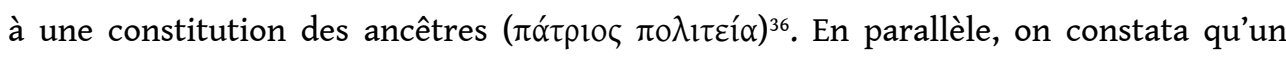
même type de constitution pouvait se révéler très varié, selon certaines particularités politiques, sociales et morales ${ }^{37}$. Thucydide découvrit quelques écarts, proches du concept moderne de « constitution réelle ${ }^{38} »$. Il s'ensuivit certaines nuances, qui furent en fin de compte systématisées dans la distinction opérée par Platon et Aristote entre les formes bonnes et mauvaises associées à chacun des trois modèles de constitutions ${ }^{39}$. Depuis le début du IV siècle, l'histoire de la théorie constitutionnelle coïncida principalement avec la recherche de nouveaux critères pour définir les constitutions, après que l'on eut à nouveau constaté combien la situation dépendait de ceux qui disposaient de l'ensemble des droits, c'est-à-dire des gouvernants, mais aussi, tout particulièrement, de la légalité du gouvernement. Socrate semble avoir été le premier à soutenir ce point de vue ${ }^{40}$.

$16 \mathrm{Au} \mathrm{V}$ siècle, un large consensus dominait, parmi les partisans et les adversaires de la démocratie, sur ses caractéristiques générales. Elle repose sur l'égalité entre riches et pauvres; en d'autres termes, tous les hommes nés de citoyens, ou du moins un très grand nombre d'entre eux, jouissent pleinement des droits politiques et sont des citoyens ${ }^{41}$. Telle est la définition première et la plus importante. La démocratie implique la liberté à l'égard du pouvoir d'un seul ou d'un petit nombre. Cette liberté se matérialise dans la domination du peuple ${ }^{42}$ [828] et se définit d'une part par le tirage au sort, d'autre part par le renouvellement annuel des dirigeants ${ }^{43}$. Toutes les décisions importantes, a minima, sont soumises à l'assemblée ${ }^{44}$. Dans cet espace, la majorité décide ; surtout, l'« iségorie ${ }^{45}$ » y prévaut - le droit de parole et de proposition dont jouit tout citoyen, expression particulièrement importante de son statut et de sa liberté, essentielle, également, pour que tout puisse être exposé librement devant le peuple. Les magistrats sont tenus de rendre des comptes ${ }^{46}$. On s'appuie sur un droit écrit. Ce dernier fut particulièrement mis en exergue par les partisans de la démocratie ; il fondait en effet l'affirmation selon laquelle la démocratie constituait le véritable État de droit et la constitution par excellence ${ }^{47}$. Enfin, chez Thucydide, dans 
l'oraison funèbre de Périclès, on insiste tout particulièrement sur le fait que les citoyens, en démocratie, peuvent vivre comme ils l'entendent et ne sont pas éduqués selon des normes figées et sous la férule d'un État surveillant leur mode de vie, comme à Sparte ou dans d'autres oligarchies ${ }^{48}$.

Les amis de la démocratie et ses ennemis nourrissaient une conception différente du

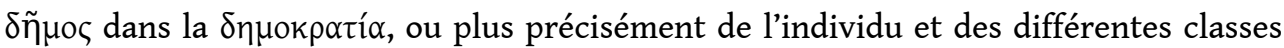
[Stände] dans cette constitution. Les uns assimilaient le $\delta \tilde{\eta} \mu о \varsigma$ au bas peuple, le trouvaient inculte et impétueux et jugeaient parfois qu'il était contrôlé par des démagogues irresponsables ${ }^{49}$. En parallèle, d'autres conceptions s'offraient à la fin du $\mathrm{V}^{\mathrm{e}}$ siècle, selon lesquelles, dans la démocratie, le peuple tout entier entre fort justement en ligne de compte. Chez Thucydide, le Syracusain Athénagoras incarne une variante de cette vision :

«Je répondrai que le mot peuple désigne la totalité des citoyens et celui d'oligarchie une fraction seulement d'entre eux, que les riches sont peut-être les plus compétents en matière de finance, mais que ce sont les gens intelligents qui donnent les meilleurs conseils et la multitude qui, une fois informée, prend les meilleures décisions. Or en régime démocratique, ces trois catégories de citoyens sans distinction, chacun dans le rôle qui lui revient ou toutes ensemble, jouissent de droits égaux..$^{50}$ "

Une autre variante envisageait davantage le citoyen particulier et insistait sur l'égalité des droits et des chances, de sorte, par exemple, que le citoyen le plus estimable, dans cette conception, devait exercer la plus grande influence: en démocratie, les classes [Stände] s'abolissaient en quelque sorte dans le champ politique ${ }^{51}$. [829] On affirmait fréquemment que l'opinion de la multitude - à la différence de celle des individus qui la composent - était juste. Ces conceptions, qui, en dehors de l'idéal et de la propagande, ont probablement dû s'enraciner dans l'image effective de nombreuses démocraties, avaient des répercussions sur l'idée courante que les démocrates se faisaient de leur constitution, comme nous pouvons le constater chez les orateurs attiques. Enfin, il convient aussi de mentionner l'opinion très répandue selon laquelle la démocratie rendait la polis plus forte vis-à-vis de l'extérieur ${ }^{52}$.

18 La théorie, pour ce que nous en connaissons, évolua surtout dans le sillage des adversaires de la démocratie. Dès lors que l'on s'interrogeait à nouveau, essentiellement, sur la nature des gouvernants, la démocratie ne pouvait guère être rangée parmi les constitutions bonnes. Il est vrai que ce jugement évolua, au plus tard avec Aristote. Même pour lui, cependant, qui connaissait une multitude de modèles de démocratie, à partir desquels il travaillait, la dernière phase de la démocratie athénienne, la démocratie radicale, dont il faisait de surcroît un portrait tendancieux, apparut comme le modèle permettant de construire une définition générale de cette constitution ${ }^{53}$.

Pour Platon, la démocratie se définissait d'abord par la liberté : « Eh bien, tout d'abord, ne faut-il pas dire que les citoyens y sont libres et que la cité laisse place à la liberté et à la libre expression? et que dans cette cité règne le pouvoir de faire tout ce qu'on veut $^{54}$ ?» Dans ce régime, rien n'est façonné selon une unité ; toutes les traditions, toutes les constitutions sont réunies dans ce "grand marché aux constitutions politique ${ }^{55}$ ». Les hommes sont « de nature diverse ». " Il s'agit apparemment d'une constitution politique agréable, privée d'un réel gouvernement [...], et qui distribue une égalité bien particulière tant aux égaux qu'à ceux qui sont inégaux ${ }^{56}$." Mais cette égalité n'est pas seulement l'expression d'une générosité en toutes choses et envers 
chacun, elle conduit aussi, dans le même temps, à un appétit farouche de liberté : « Les gouvernants qui passent pour des gouvernés, et les gouvernés qui passent pour des gouvernants, ce sont eux dont on fait l'éloge en privé comme en public, ce sont eux auxquels on accorde du respect ${ }^{57}$." Ainsi, Platon constatait, là où Aristote devait déceler une transformation des gouvernants et des gouvernés, une assimilation dans leurs rôles respectifs. Il évoquait plus loin le fait que le fils se fait l'égal du père, la femme de l'homme, le métèque du citoyen, l'esclave du maître et même l'animal de $l^{1} h o m m e^{58}$. Son véritable intérêt se portait sur l'homme démocratique. Il prétendait en effet qu'à chaque constitution correspond un type d'homme. "Ou alors crois-tu que les constitutions politiques surgissent "des chênes ou des rochers", et non des caractères de ceux qui habitent dans les cités, lesquels, comme lorsque les poids de la balance se renversent, finissent par entraîner tout le reste du côté où ils penchent ${ }^{59}$ ? "Chez Platon, l'homme démocratique ne se définit pas par son rang social [ständisch], ses convictions, sa sensibilité ou ses intérêts, mais par sa nature même ; et celle-ci n'est pas déterminée en fonction de la constitution, elle se forme, au contraire, d'une manière ou d'une autre, en même temps qu'elle, la succession des constitutions étant régie par une loi historique ${ }^{60}$ [830] selon laquelle toute constitution est bientôt promise à un inéluctable déclin aussi longtemps que l'on ne l'abandonne pas pour en créer une autre, idéale. L'homme démocratique fait toujours ce qu'il lui plaît, accorde une valeur égale aux bons et aux mauvais désirs et se laisse dominer par eux, aussi arbitrairement que le tirage au sort au moyen duquel la cité attribue les magistratures ${ }^{61}$.

Dans le Politique, Platon distingue - le premier, semble-t-il - une bonne et une mauvaise démocratie. (On avait déjà distingué auparavant la royauté et la tyrannie, ainsi que l'aristocratie et l'oligarchie ${ }^{62}$. Il se peut également que l'on ait considéré la " constitution (mixte) des ancêtres" comme une bonne démocratie ${ }^{63}$.) Toutefois, il estime que la différence entre ces deux régimes n'est pas si grande. "Pour [le gouvernement] de la multitude, écrit-il en effet, tout y est faible et il ne peut rien faire de grand, ni en bien, ni en mal, comparativement aux autres, parce que l'autorité y est répartie par petites parcelles entre beaucoup de mains ${ }^{64}$. . C'est bien la raison pour laquelle il ne réserve pas un nom spécifique à ces deux formes de démocratie. Ce qui fonde la différence, c'est la question de savoir si la domination est exercée conformément aux lois ${ }^{65}$. Étant donné cependant que, dans la démocratie - comme dans la monarchie, la timocratie, l'oligarchie, etc. -, les gouvernants et les gouvernés se comportent comme des maitres et des esclaves, elle mérite aussi peu le nom de

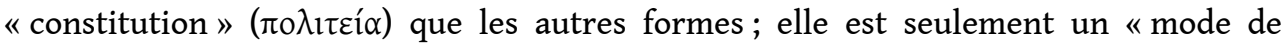

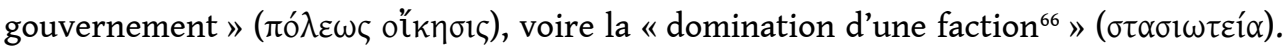
Elle se développe à partir de l'oligarchie et donne naissance à la tyrannie ${ }^{67}$.

21 Vers le milieu du IV ${ }^{e}$ siècle, pour désigner la bonne forme de la démocratie, on utilisa

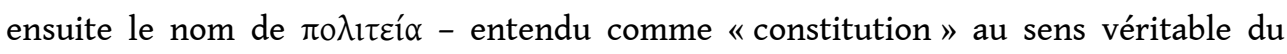
terme ou bien comme "État constitutionnel». Même Aristote l'emploie

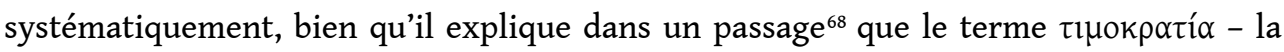
domination d'un groupe, relativement large, de citoyens aisés - serait plus adapté. On reconnaissait ainsi la prétention de la démocratie à s'assimiler à la $\pi 0 \lambda_{\imath \tau \varepsilon i ́} \alpha^{69}$, à l'heure où, dans la théorie, ce terme ne renvoyait plus seulement à la " communauté civique " (ou à l'« État des citoyens »), mais à la « constitution », en tant qu'elle représentait une organisation juridique et un «État constitutionnel ». Dans le même temps, on limitait cette prétention à la bonne variante de la démocratie, qui était en réalité une sorte de 


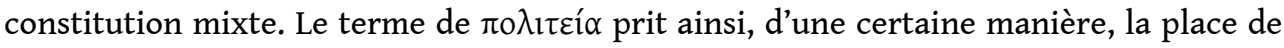

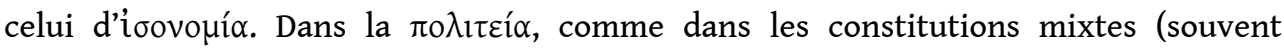

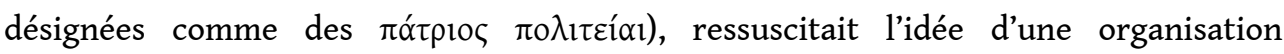
garantissant réellement l'égalité et le droit, qui avait été abandonnée après l'exacerbation de la notion de pouvoir.

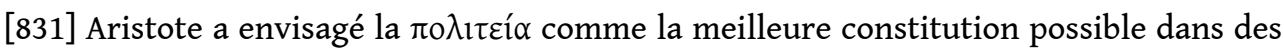
conditions normales ${ }^{70}$. Il la définit comme un mélange d'oligarchie et de démocratie, ou plus précisément comme la version de ce mélange tendant vers la démocratie, l'autre version étant l'aristocratie. Le mélange doit être si parfait qu'il est possible de tenir cette constitution autant pour une démocratie que pour une oligarchie, ou bien encore

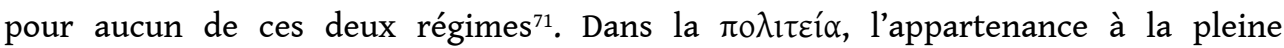
citoyenneté présuppose certes un cens; mais celui-ci n'est pas si élevé - comme dans les oligarchies - qu'une grande partie ou même, de préférence, la majorité des hommes

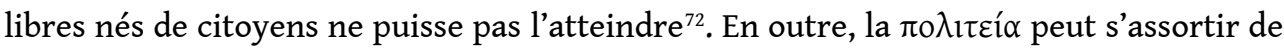
ces dispositions particulières, très répandues dans les oligarchies ou les démocraties, visant à garantir ou à entraver, selon les cas, la participation des citoyens pauvres ou fortunés ${ }^{73}$. Les principes définissant la composition du corps civique, en particulier, deviennent ainsi mixtes, ainsi que les institutions correspondantes. À l'inverse, le contrôle mutuel ou les interactions entre des organes reposant sur différentes classes [Stände], ne jouent qu'un rôle limité. Conformément à la réalité d'alors, Aristote connaissait surtout la domination [Herrschaft] directe de l'ensemble du corps civique, exercée par le biais de commissions ou de magistrats. La question déterminante était donc seulement de savoir qui composait le corps civique.

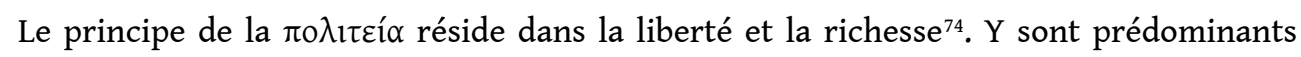
ceux du milieu (en gros, les hoplites) ${ }^{75}$. Ce sont les meilleurs citoyens. Ils sont le mieux à même d'obéir à la raison, ils peuvent combler le fossé entre riches et pauvres et pourvoir aux besoins de la communauté, alors que les autres ne veulent que dominer ou (en partie) servir ${ }^{76}$. «La cité veut être le plus possible composée de citoyens égaux et semblables, comme le sont, plus que les autres, ceux du milieu. La cité est donc gouvernée au mieux lorsqu'elle est composée de citoyens dont nous avons dit qu'ils constituent par nature une cité ${ }^{77}$.» Une telle cité repose sur "une constitution commune et véritablement égale ${ }^{78} "$, en d'autres termes, elle répartit l'égalité selon la dignité. Et comme les affaires communes renvoient dans le même temps aux affaires de la cité, au " politique ", cette constitution - c'est ainsi qu'il conviendrait de compléter

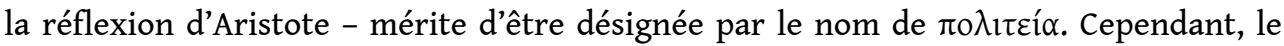
texte transmis indique seulement: "Quand c'est la multitude qui détient le gouvernement en vue de l'avantage commun, la constitution est appelée du nom commun à toutes les constitutions, à savoir $\pi 0 \lambda_{\imath} \tau \varepsilon i \alpha^{79}$. "

24 Alors que la monarchie idéale et l'aristocratie reposaient essentiellement sur la qualité, acquise par l'éducation, d'un seul ou d'un petit nombre, la forme idéale de la démocratie ne pouvait reposer que sur l'estime, apparue depuis la fin $\mathrm{du} \mathrm{V}^{\mathrm{e}}$ siècle, envers les classes intermédiaires, autrement dit sur l'hypothèse que la qualité [832] des

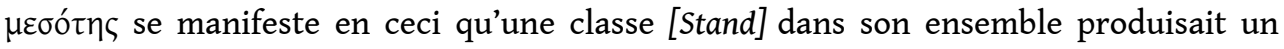
éthos particulier en raison de sa position économique. Comme les classes

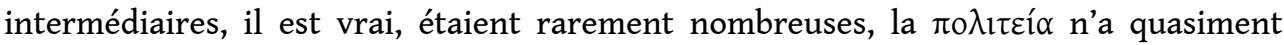
jamais existé ${ }^{80}$ et, même dans le système que forme la théorie aristotélicienne des 
constitutions, elle n'a été qu'insuffisamment prise en compte - indépendamment du fait qu'elle glisse presque imperceptiblement vers les formes voisines.

L'autre forme de démocratie qui s'écarte de la norme est définie par Aristote, ainsi que nous l'avons mentionné, comme une constitution dans laquelle les hommes libres et pauvres, formant la majorité, détiennent le pouvoir suprême. Cette situation implique que ces derniers exercent leur domination selon leur propre avantage, et non dans l'intérêt de la communautés ${ }^{8}$. Aristote voyait lui aussi dans la liberté le principe de la démocratie : dès lors que la démocratie se définit par l'égalité, elle tire son contenu de la liberté ${ }^{82}$. L'égalité démocratique est en effet une égalité fondée sur le nombre (et non sur la valeur). « Un régime populaire, en effet, naît du fait que des gens qui sont égaux dans un domaine estiment être égaux absolument: c'est parce qu'ils sont tous pareillement libres qu'ils estiment être égaux absolument ${ }^{83}$. » La « liberté » signifiait ici tout d'abord le statut de personne en tant qu'il est la qualification minimale requise pour la pleine citoyenneté. Au-delà, elle est constituée avant tout par deux éléments : les citoyens sont tour à tour gouvernants et gouvernés, d'une part, et ils vivent, d'autre part, comme ils l'entendent ${ }^{84}$. Cette situation découle de l'égalité fondée sur le nombre, car si cette égalité constitue l'équité démocratique, les hommes libres (et notamment les pauvres) doivent pouvoir prendre part aux magistratures, et ce de la manière la plus uniforme possible ${ }^{85}$. De même, la liberté, en tant que liberté par rapport à la domination, dans la mesure où celle-ci est l'unique qualification du citoyen de plein droit, consiste en cette alternance. Elle est ainsi conçue moins comme un droit contre le gouvernement que comme un droit de gouverner. Dans un autre passage, Aristote décrit la démocratie par le fait que tous ont autorité sur toutes choses et que la majorité (des hommes libres) décide ${ }^{86}$. Le fait que le pouvoir de l'assemblée est rendu possible, sur le plan institutionnel, par le renouvellement permanent des magistratures et la désignation récurrente, par tirage au sort, des magistrats et des membres du conseil, ne joue chez Aristote aucun rôle, bien qu'il mette en exergue ces deux facteurs envisagés comme des dispositions démocratiques. La liberté de mener sa vie comme on l'entend renvoie surtout à une liberté vis-à-vis de la tutelle exercée par les organes d'éducation et de surveillance des mœurs, autrement dit à une limitation des prérogatives de l'État. «De là est venue la revendication de n'être, au mieux, gouverné par personne, ou sinon de l'être à tour de rôle. Et cela va dans le sens de la liberté fondée sur l'égalité87. »

$\mathrm{Au}$ sein de la démocratie, Aristote distingue une série de particularités, en utilisant comme critères la composition du corps civique, d'une part, [833] et la question du kúprov, du pouvoir suprême, d'autre part, qui vise plus précisément à déterminer s'il revient aux lois - elles sont alors, bien entendu, démocratiques - ou à l'assemblée dont on suppose dans ce cas qu'elle ne respecte pas les lois. Pour Aristote, ces deux critères entretiennent un rapport étroit, la dimension juridique n'étant guère plus qu'une fonction de la dimension sociale ${ }^{88}$. L'élément décisif pour appréhender la nature d'une démocratie consiste ainsi à déterminer quelle strate de la population prédomine et de quelle manière - en principe selon un cens, au mieux peu élevé - l'accès à la pleine citoyenneté est organisé. Là où les paysans, par exemple, et d'autres couches sociales dominent moyennant un cens modéré, dans les meilleures démocraties, on n'a guère le temps d'organiser d'autres assemblées que celles qui sont indispensables - on laisse donc les lois s'appliquer d'autant plus volontiers. Dans un tel régime, ce sont aussi les meilleurs citoyens qui gouvernent, car seul celui qui possède suffisamment d'argent et de temps est en mesure d'exercer les magistratures. Dans la démocratie 
radicale, à l'inverse, l'artisan et l'ouvrier salarié se voient verser des indemnités ; les pauvres peuvent ainsi prendre part aux assemblées qui se réunissent fréquemment et exercer des magistratures. Les décrets du peuple dominent, et les démagogues exercent une influence décisive. Les différences institutionnelles ne jouent tout au plus un rôle qu'en fonction des différences sociales ou civiques. Ainsi, dans une démocratie où seul un nombre limité d'assemblées peut être tenu, le conseil représente l'organe démocratique le plus important, tandis que cette instance, dès lors que des indemnités sont versées, est largement dépossédée de son pouvoir ${ }^{89}$. Dans la dernière phase de la démocratie, la démocratie radicale, le peuple exerce dans les faits une domination despotique. On peut prétendre qu'elle n'est pas une constitution - gouvernée par les lois - et qu'elle n'est donc pas une démocratie ${ }^{90}$. Toutefois, c'est à la démocratie extrême qu'Aristote emprunte l'un des deux traits de la liberté démocratique, à savoir la possibilité de vivre comme on veut ${ }^{91}$. De même, l'alternance entre gouvernés et gouvernants n'est pas partout présente, mais presque toujours. À défaut, la pratique de l'élection et l'obligation de rendre des comptes à l'assemblée plaident en faveur du caractère démocratique de la constitution. Dans tous les cas, la participation de tous ou de presque tous les hommes libres constitue la marque distinctive de la démocratie.

Si Aristote opéra une distinction entre des formes premières et des formes tardives prises par la constitution, et surtout, par la démocratie, il était clair pour lui que sans les transformations historiques, et à plus forte raison de la société et de l'organisation militaire, les diverses formes de constitutions n'auraient pas été possibles. Il ne prévoyait pas cependant que l'histoire de la constitution poursuive son évolution dans une certaine direction ; il estimait au contraire que, du fait de la taille acquise par les cités, la constitution démocratique était appelée à durer. Il connaissait un cycle des constitutions, mais n'attendait pas des mutations sociales. Pour lui, dans la hiérarchie des constitutions, la démocratie, puis l'oligarchie et la tyrannie suivaient la politie ${ }^{92}$. La monarchie et l'aristocratie était certes préférables, mais elles n'étaient possibles que dans certaines conditions.

Après Aristote, pour autant que nous puissions en juger, la politie, comme forme bonne de la démocratie, [834] n'a plus joué aucun rôle. Polybe désigne la forme correcte par le terme "démocratie " et réserve de nouveaux noms à la forme déviante : óx入okpatía,

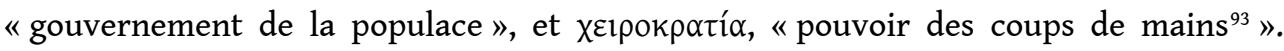
Selon lui, on peut parler de démocratie « là où les citoyens, respectueux de la coutume et de la tradition, vénèrent les dieux, honorent leurs parents, respectent leurs anciens et obéissent aux lois, là où d'autre part la volonté de la majorité est souveraine ${ }^{94}$ ». La démocratie se développe à partir de l'oligarchie et se maintient aussi longtemps que l'égalité et la liberté ne vont pas de soi, c'est-à-dire durant deux générations. Elle se transforme ensuite en une ochlocratie, étant donné que la troisième génération devient excessive dans son ambition de puissance et que le peuple est habitué à recevoir des présents, de sorte qu'il tombe sous la dépendance d'un chef qui instaure une domination violente ${ }^{95}$. Comme aux yeux de Platon, les critères moraux et l'enchaînement qui sous-tend à partir de ce moment l'évolution des constitutions, presque régie selon des lois, sont pour Polybe déterminants. Ce dernier n'envisage pas plus avant la structure sociale. Dans l'intervalle, les grands avantages de l'approche aristotélicienne avaient été oubliés. Pour Polybe, seule était durable la constitution mixte, qu'il avait lui-même - ou l'un de ses prédécesseurs - définie selon l'idée nouvelle d'un système fondé sur l'équilibre entre divers facteurs relativement autonomes. 

II siècle av. J.-C., le sens général de "constitution libre", par opposition à la monarchie. Il désignait donc des démocraties tout autant que des oligarchies (du moins nombre d'entre elles) et devint en partie synonyme de "liberté", y compris par rapport à des puissances extérieures ${ }^{96}$. Il s'avère difficile de déterminer comment on est parvenu à cette situation. Vraisemblablement, la différence avec les puissantes monarchies de cette période l'emportait tellement que l'on était indifférent à tout autre distinction. L'intérêt des couches les plus modestes pour la politique s'était aussi si affaibli que l'on pouvait gouverner de manière oligarchique dans le cadre de formes démocratiques. De tels régimes se faisaient ainsi largement passer pour des démocraties. Dans les premiers siècles de l'ère chrétienne apparut un autre glissement intéressant du concept: Aristide décrit l'Empire romain comme une "démocratie commune à la terre " instaurée "sous l'autorité unique du meilleur gouvernant et ordonnateur ${ }^{*} »$. Dion Cassius, pour sa part, estime que la véritable démocratie s'épanouit sous la monarchie. Dans sa forme habituelle, elle s'apparente selon lui à la liberté de la populace et à l'asservissement des meilleurs, et donc à la corruption générale. Dans le premier cas, à l'inverse, on honore la modération et on accorde à chacun une part égale selon sa dignité. Dans cette perspective, le but idéal de la constitution supplanta les institutions qui devaient garantir la réalisation de cet objectif. L'enjeu ne résidait plus dans les organes de la constitution, mais dans la (prétendue) réussite des pratiques de gouvernement ; [835] et si cette réussite résidait dans la liberté - comprise comme garantie sociale et juridique -, alors on avait justement affaire à une démocratie ${ }^{97}$. Le terme n'apparaît pas en latin, hormis dans trois passages isolés. Dans le De re publica, Cicéron évoque un État populaire (où toute chose réside dans le peuple). Il différencie le bon État populaire (le peuple juste et modéré) du mauvais État populaire (où règne le tourbe et l'anarchie) ${ }^{98}$. Il empruntait ainsi ses vues sur la question à la théorie grecque (pour Rome, voir l'article «République » dans le volume 5 des Geschichtliche Grundbegriffe).

(Christian Meier)

\section{BIBLIOGRAPHIE}

Berve, Helmut (1953) : Die Herrschaft des Agathokles, Munich.

Busolt, Georg (1920) : Griechische Staatskunde, $3^{\mathrm{e}}$ éd., Munich.

Diels, Hermann / Kranz, Walther (éd.) (1966) : Die Fragmente der Vorsokratiker, $12^{\mathrm{e}}$ éd., Dublin / Zurich.

Fuks, Alexander (1953) : The Ancestral Constitution, Londres.

Grossmann, Gustav (1945/1950) : Politische Schlagwörter aus der Zeit des Peloponnesischen Krieges, thèse de doctorat, Bâle /Zurich.

Henkel, Hermann (1872) : Studien zur Geschichte der griechischen Lehre vom Staat, Leipzig. 
Jones, Arnold H. M. (1957) : Athenian Democracy, Oxford.

Larsen, Jakob A. O. (1945) : «Representation and Democracy in Hellenistic Federalism », Classical Philology, 40, p. 65-97.

Larsen, Jakob A. O. (1948) : « Kleisthenes and the Development of the Theory of Democracy at Athens ", in : Konvitz, Milton R. / Murphy, Arthur E. (éd.) : Essays in Political Theory, Presented to G. H. Sabine, Ithaka, p. 1-16.

Larsen, Jakob A. O. (1954) : « The Judgement of Antiquity on Democracy », Classical Philology, 49, p. 1-14.

Meder, Anton (1938) : Der athenische Demos zur Zeit des Peloponnesischen Krieges im Lichte der zeitgenössischen Quellen, thèse de doctorat, Munich.

Meier, Christian (1968) : « Drei Bemerkungen zur Vor- und Frühgeschichte des Begriffs Demokratie », in : Discordia Concors (Festschrift für Edgar Bonjour), vol. I, Bâle, p. 3-29.

Meier, Christian (1970) : Entstehung des Begriffs « Demokratie ». Vier Prolegomena zu einer historischen Theorie, Francfort-sur-le-Main.

Romilly, Jacqueline de (1959) : «Le classement des constitutions d'Hérodote à Aristote », Revue des études grecques, 72, p. 81-99.

Vlastos, Gregory (1953) : « Isonomia », American Journal of Philology, 74, p. 337-366.

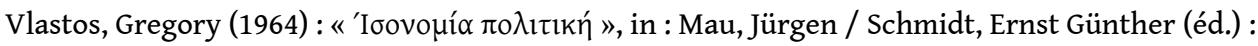
Isonomia. Studien zur Gleichheitsvorstellung im griechischen Denken, Berlin, p. 1-35.

\section{NOTES}

1. Meier (1968), p. 5 et note 3, p. 24. Pour son analyse dans un cadre complet, voir Meier (1970), p. 7 sq.

2. Meier (1968), p. 26-27.

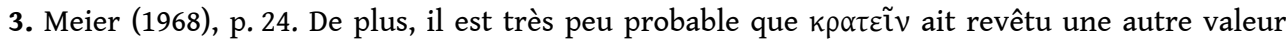
qu'äpxzıv. Chez Hérodote, dans la discussion sur les constitutions introduisant de fines nuances sur le plan de la terminologie, les partisans comme les adversaires utilisent le terme d'ö $\rho x \varepsilon ı$

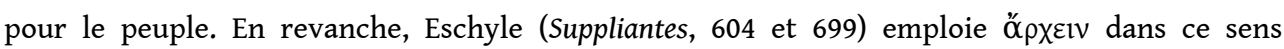
assurément positif (cf. Euripide, Cyclope, 119). Voir en parallèle Meier (1970), p. 45 sq.

4. Meier (1968), p. 18 sq. ; (1970), p. 40.

5. Pindare, Pythiques, II, 86 sq. (trad. A. Puech).

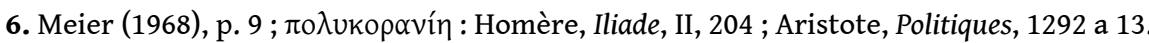

7. Meier (1968), p. 9.

8. Sur celle-ci, voir Meier (1968), p. 5 sq. ; (1970), p. 15 sq.

9. Sur ce point, voir en particulier Hérodote, III, 80, 5-6.

10. Vlastos (1964), p. 1 sq.

11. Meier (1968), p. 20 sq. ; (1970), p. 40.

12. Formule attestée pour la première fois chez Eschyle, Suppliantes, 604 et 699, une œuvre probablement composée en 464 av. J.-C.

13. Euripide, Suppliantes, 405-406 (trad. M. Artaud).

14. Meier, (1970), p. 40 sq.

15. Thucydide, III, 62,4 .

16. Hérodote, III, 80, 6, ainsi que Vlastos (1953), p. 358. Il convient en particulier de mentionner l'obligation de reddition des comptes des magistrats : voir Larsen (1948), p. 7 et p. 9 sq. 
17. Hérodote, III, 80, 6 [N.d.T. : nous adaptons ici la traduction de A. Barguet, Christian Meier évoquant, à la fin de la citation d'Hérodote, non pas la «majorité », mais l'« ensemble »

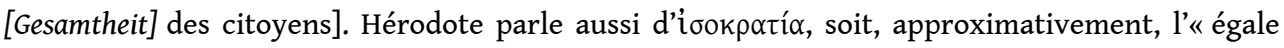

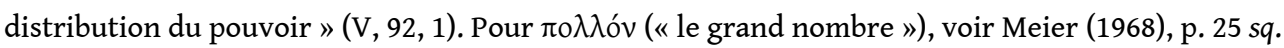
sur le terme correspondant de $\pi \lambda \tilde{\eta} \theta$ os.

18. Vlastos (1964), p. 9.

19. Même si, naturellement, il resta possible, comme auparavant, de différencier l'zủvouía et

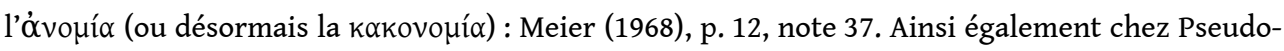
Xénophon, Constitution des Athéniens, I, 8.

20. Attesté pour la première fois dans la discussion sur les constitutions chez Hérodote, III, $80 \mathrm{sq}$.

21. Voir Pseudo-Xénophon, Constitution des Athéniens, I, 5-6 et 8-9; III, 10 ; Thucydide, III, 82.

22. Ainsi déjà chez Hérodote, III, 82, 4; Aristote, Politiques, 1302 a 8, ainsi que dans plusieurs autres passages.

23. Ce qui s'est traduit, dans une proportion presqu'invraisemblable, par une multitude de renversements constitutionnels.

24. Voir Pseudo-Xénophon, Constitution des Athéniens, I, 1 sq.

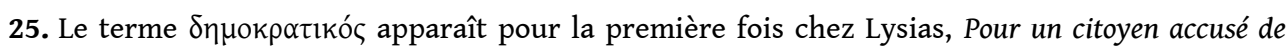
menées contre la démocratie, 8 : « Avant tout, posons pour principe, que nul homme par caractère n'est partisan de l'état oligarchique ou démocratique: chacun désire de changer de gouvernement suivant son intérêt. » (trad. A. Auger).

26. Pseudo-Xénophon, Constitution des Athéniens, I, 7.

27. Pseudo-Xénophon, Constitution des Athéniens, III, 9.

28. Aristote, Politiques, 1279 b 11 sq.

29. Aristote, Politiques, 1290 b 17 (trad. P. Pellegrin).

30. Grossmann (1945/1950), p. 79-80 ; Meder (1938), p. 102 sq., en particulier p. 106-107.

31. Voir, outre le Pseudo-Xénophon, Thucydide, II, 36 sq. Sur les lois : Thrasymaque chez Platon, République, $338 \mathrm{~d}$ sq. et Lois, 714 b. La découverte de la diversité des vónot est ancienne (Hérodote, III, 38). Désormais, ils étaient liés pour la première fois à l'avantage des gouvernants.

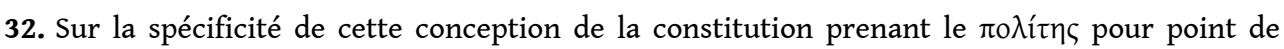
départ et se substituant à d'autres conceptions, en tant qu' « institution », « ordre » : voir Meier (1970), p. 57 sq.

33. Meier (1970), p. 56-57.

33a. 33a Pseudo-Xénophon, Constitution des Athéniens, passim, en particulier, I, 7 ; Thucydide, III, 82,8 , parmi d'autres passages.

34. Voir les témoignages (se rattachant aux exagérations de la comédie) réunis chez Meder (1938), p. 38-39. Les témoignages sont par la suite très nombreux chez Platon et Aristote.

35. Voir Aristote, Politiques, 1296 a $22 \mathrm{sq}$.

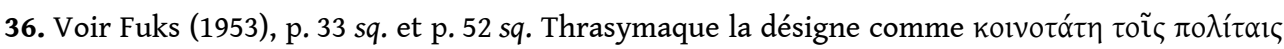

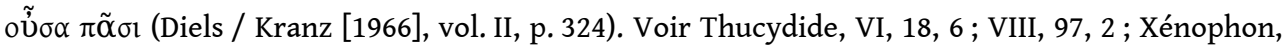
Helléniques, II, 3, 48 ; Grossmann (1945/1950), p. 24-25.

37. Thucydide, III, 62 , 3 ; IV , 74, 3 ; V, 81, 2 ; VIII, 53, 3 ; VIII, $89,2$.

38. Thucydide, II, $65,9$.

39. Voir Romilly (1959), p. $81 \mathrm{sq.}$

40. Xénophon, Mémorables, IV, 6, 12. Voir Henkel (1872), p. 44-45. Voir aussi Isocrate, Panégyrique, $132 \mathrm{sq}$.

41. Cf. l'iøovouía : Hérodote, III, 80, 6 ; Euripide, Suppliantes, 407-408; Thucydide, II, 37.

42. Euripide, Suppliantes, 405-406 ; Pseudo-Xénophon, Constitution des Athéniens, I, 8 : Le dèmos ne veut pas être asservi, mais « être libre et dominer ». Le « peuple » renvoie chez ces deux auteurs à un contenu très différent. 
43. Hérodote, III, 80, 6 ; Euripide, Suppliantes, 406-407.

44. Hérodote, III, 80, 6 .

45. Hérodote, V, 78 ; Euripide, Suppliantes, 438 sq. ; Pseudo-Xénophon, Constitution des Athéniens, I, 2, 6. Busolt (1920), vol. II, p. 418-419.

46. Voir supra, note 16.

47. Euripide, Suppliantes, 430 sq. ; Eschine, I, 4-5 ; Démosthène, VI, 25 ; 24, 75-76 ; Hypéride, Pour

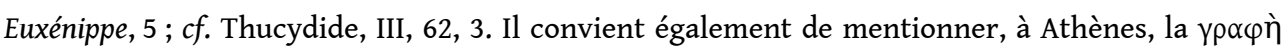
$\pi \alpha \rho \alpha v o ́ \mu \omega v$ et la distinction entre vó $\mu$ ol et $\psi \eta \varphi$ í $\sigma \alpha \alpha \alpha$ : sur ce point, voir Jones (1957), p. 53. Cf. infra, note 69.

48. Thucydide, II, 37. Cf. Grossmann (1945/1950), p. 83 ; Meder (1938), p. 44.

49. Hérodote, III, 81-82 ; Euripide, Suppliantes, 416 sq. Cf. Pseudo-Xénophon, Constitution des Athéniens, I, 7 ; Meder (1938), p. 130 sq. Typique des opposants : les objections pratiques. Pour les réponses : l'ethos démocratique (Euripide).

50. Thucydide, VI, 39, 1 (trad. D. Roussel). Hérodote (III, 80) évoquait également «le pouvoir du peuple tout entier ». Voir Meier (1968), p. 25 sq.

51. Thucydide, II, 40, 2 ; VI, 39, 1 ; Platon, Protagoras, 323a. À une époque ultérieure : Aristote, Politiques, 1281 a 40 sq.; b 34 ; 1282 a 16, entre autres passages. Larsen (1954), p. 3-4 ; Meder (1938), p. 123-124.

52. Hérodote, V, 78 ; 91 ; Thucydide, II, 36, 3-4. Cf. Euripide, Suppliantes, 442 sq.

53. En quoi Aristote, par exemple, omet que, dans son modèle, les lois règnent entièrement à Athènes.

54. Platon, République, 557b (trad. G. Leroux).

55. Ibid., 557d.

56. Ibid., 555c.

57. Ibid., 562d.

58. Ibid., 563.

59. Ibid., 544d-e.

60. Ibid., 555b sq. ; 559d sq.

61. Ibid., 561.

62. Socrate chez Xénophon, Mémorables, IV, 6, 12 (à la place de l'«oligarchie»: la «ploutocratie »).

63. La position de l'une à l'égard de l'autre n'est pas tout à fait claire. Il se peut qu'on l'ait considérée comme une forme particulière de démocratie, mais aussi, fréquemment, comme un quatrième type de constitution (cf. par exemple Isocrate, Panathénaïque, 131-132), pour autant que l'on s'en soit rendu compte.

64. Platon, Politique, 302d (trad. É. Chambry) ; 303a.

65. Ibid., 302e. Ailleurs : Lois, 832c : « Le pouvoir seul est volontaire, et l'obéissance est toujours forcée. " (trad. V. Cousin).

66. Platon, Lois, 712e ; 715b; 832c. Cf. Politique, 303c.

67. Platon, République, 555b sq.; 562a sq. ; 565d ; 566c sq. ; 569c.

68. Aristote, Éthique à Nicomaque, 1160 a 34.

69. Démosthène, I, 5 ; VI, 21 ; VI, 25 ; XV, 20 ; Isocrate, IV, 125 ; Lettres, VI, 11. Cf. supra, note 47.

70. Aristote, Politiques, 1295 b 34. Cf. 1295 a 25 sq.

71. 1265 b $26 ; 1293$ b $33 ; 1294$ b $13 ; 1294$ b $34 ; 1307$ a 15 .

72. $1297 \mathrm{~b} 1$. Cf. Éthique à Nicomaque, $1160 \mathrm{~b} 18$.

73. Aristote, Politiques, 1294 a 35 sq.

74. 1294 a 20.

75. 1297 b 1. Cf. 1306 b $11 ; 1321$ a $12 ; 1302$ a 13.

76. 1295 a. 37 sq. Voir aussi 1292 b 25 ; 1293 a 12.

77. 1295 b 25 (trad. mod. P. Pellegrin). 
78. 1296 a 29 (trad. mod. P. Pellegrin). Cf. 1286 b $13 ; 1297$ a 2.

79. 1279 a 37. Cf. Meier (1970), p. 63 et p. 78.

80. Aristote, Politiques, 1293 a $40 ; 1296$ a $23 ; 1296$ a 36.

81. 1279 b $8 ; 1284$ b 5 sq.

82. 1291 b 34 , entre autres passages.

83. 1301 a $28 ; 1301$ b 29 sq. Cf. 1280 a $7 ; 1280$ a 24 .

84. 1317 b 2 .

85. 1299 b $26 ; 1309$ a 2 .

86. 1310 a 28 . Cf. 1291 b $37 ; 1298$ a 9.

87.1317 b 13.

88. 1292 b 22 sq. ; 1318 b 6 sq.

89. 1317 b $30 ; 1323$ a $4 ; 1299$ b 38 .

90. 1292 a 34. Cf. 1312 b $5 ; 1312$ b 35.

91. 1310 a 25 .

92. $1289 \mathrm{~b} 4 ; 1296 \mathrm{~b} 3$ sq. ; Éthique à Nicomaque, $1160 \mathrm{~b} 20$.

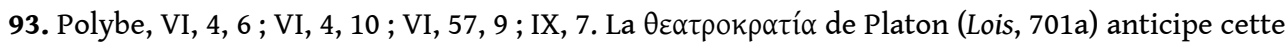
lecture. Polybe et Philon d'Alexandrie (Des vertus, 180) sont les deux seuls auteurs connus à opérer une distinction entre les bonnes et les mauvaises démocraties, en réservant ce nom aux premières. Durant la période hellénistique et à l'époque impériale, le terme avait manifestement très bonne presse. Pour Philon, voir encore Que Dieu est sans changement, 176; D'Abraham, 242 ; De la confusion des langues, 108.

94. Polybe, Histoires, VI, 4, 5 (trad. A. Barguet).

95. VI, 8, 4 sq.

96. Larsen (1954), p. 9-10 ; (1945), p. 88-89; Berve (1953), p. 76, note 72.

*. * N.d.T. : Ælius Aristide, En l'honneur de Rome, 60 (trad. L. Pernot). Christian Meier restitue ici l'analyse d'Ælius Aristide au moyen d'une expression unique, en décrivant l'« empire universel » [Weltreich] de Rome comme une "démocratie placée sous l'autorité de l'empereur»[eine Demokratie unter dem Kaiser].

97. Cf. Jochen Bleicken, « Der Preis des Aelius Aristides auf das römische Weltreich », Nachrichten der Akademie der Wissenschaften in Göttingen, philologische-historische Klasse, 1966, p. 239, p. 250, note 54, p. 252-253 et p. 269. L'interprétation du rêve de Nabuchodonosor chez Hippolyte est particulièrement intéressante pour saisir la conception de l'époque impériale (voir Santo Mazzarino, La fin du monde antique. Avatars d'un thème historiographique, trad. A. Charpentier, Paris, Gallimard, 1973, p. 37).

98. Cicéron, De la République, I, 42 ; I, 43 ; I, 44 ; I, 69 : «civitas popularis (in quo in populo sunt omnia) »; « populus iustus et moderatus »; « furor multitudinis licentiaque ».

\section{INDEX}

Schlüsselwörter : Demokratie, Antike

Mots-clés : démocratie, antiquité 


\section{AUTEURS}

\section{CHRISTIAN MEIER}

Christian Meier est professeur émérite d'histoire ancienne à l'Université de Munich. A l'époque de la publication de ce texte, en 1972, Christian Meier était professeur à l'Université de Cologne. Pour plus d'informations, voir le lien suivant. 